\title{
The Semantic Classification in the Temporal Theory of Psychoanalysis
}

\author{
Antal F. Borbely, M.D. \\ 675 West End Ave. Apt. 2A \\ New York, NY 10025
}

\section{INTRODUCTION}

Many aspects of classification have been researched but the laws underlying changing classifications need to be focussed on. Psychoanalysis is a science dealing with the changing classifications within the individual. Aided by its theory, evolving over the last hundred years, psychoanalysis undertakes to provide actual scientific guidance in a person's changing his or her classifications or priorities in life. Such changes regularly occur in a psychoanalysis where, in the analytic setting, an analyst and an analysand form a unique relationship, usually lasting several years, for the purpose of analyzing the analysand's life. The analysand discovers his or her unique epistemologic categories as they developed based on a unique personal history. These

epistemologic categories reflect both biographic achievements as well as constraints and, usually for the first time, become accessible for both understanding and change. While understanding and change can and does, to some extent, occur outside psychoanalysis, the psychoanalytic setting provides a unique opportunity to exclusively and systematically focus on such understanding and change.

The way the analysand classifies his or her life in terms of motivations, biographic events, plans, symptoms, fears and wishes undergoes a profound and non - predictable change in the course of an analysis. One's priorities change, developmental arrest in certain domains is overcome so that psychological development can resume, one's concepts change with each insight a little and, after a cumulative process, nothing quite means what it once meant.

Hundreds of thousands of persons have by now undergone an analysis and the literature concerning psychoanalysis may number more than one million books and journals. Few other sciences have as profoundly influenced human culture in the last hundred years. While psychoanalysis suffered for a long time from a self misunderstanding of being mainly a biological science because of the great importance it accorded to sexual and aggressive drives, it deserves to be viewed as an information science par excellence. This paper attempts to justify this claim and thus to contribute towards bridging psychoanalysis and information science. Specifically, it will use the temporal theory (Borbely, 1987) to touch on classification research connected problems, for example concept acquisition, the basis for semantic classes, knowledge representation schemes, reasoning with classification schemes, compatibility between classification schemes, information retrieval, parallel processing, distributive processing, and hybrid reasoning (Frisch, 1991).

\section{THE PSYCHOANALYTIC SETTING:}

The analysand is lying in the supine position on the couch while the analyst sits behind him or her, out of the analysand's sight. The analysand free associates, that is, verbalizes whatever comes to mind without censoring it, while the analyst listens and interprets the analysand's productions. By following the "basic rule" to free associate the usual dialogue between two subjects is suspended. 
One reason for this arrangement is to allow for important communications, often unknown to the analysand, to emerge. This becomes possible by suspending the usual classifications for dialogue, for logical communication, for communication constrained by consideration for the other person's feelings or decorum.

The analyst's interpretations allow for growth promoting insights on the part of the analysand to occur based on the fact that the analyst, having undergone an analysis him or herself, does not shy away from what causes anxiety to the analysand. The analyst can therefore lift the analysand's communications to a higher, more mature classification level. Unconscious childhood conflicts involving important childhood figures surface and are reexperienced in the relationship with the analyst, to whom feelings and role assignments are transferred. The latter endeavors to remain neutral (value tolerant) and abstinent (not gratifying his or her own needs), so as to be able to show the analysand the "transference" in a convincing, insightful way. What appeared to the analysand to be an experience in the present, turns out to be a dramatization of past constellations which now, for the first time, can be understood and resolved in terms of adult categories of thinking and experiencing (Reclassification!).

Such understanding is not a mere intellectual one, as it is occurring within an experiential field containing many of the emotions of the original childhood setting. This allows for a resolution of old conflicts and a reintegration of its elements into the mainstream of psychological development leading not only to symptom removal but, more importantly, to a general creativity enhancement.

\section{SOME ELEMENTS OF GENERAL PSYCHOANALYTIC THEORY:}

Psychoanalytic theory has passed through several stages in these hundred years and continues to develop. While this development can be described from different points of view (different classification schemes can serve for the purpose of a historic overview) I chose as the most fruitful classification scheme for the history of psychoanalytic theory the one deriving from the concepts "defense" and "defended against."

One of the most basic clinical findings by psychoanalysis was that the human mind is conflicted and that often the real causes for the conflicts are, at first, unknown both to analyst as well as analysand. At first S. Freud (1900) thought that all defense was synonymous with repression. By "repression" he meant the making unconscious of what once was conscious in order to avoid anxiety. Later, Freud described other defense mechanisms: projection, introjection, undoing, rationalization, displacement, denial, isolation (of thought from affect) and others. All defenses served the purpose of warding off anxiety by psychologically severing the realistic connections between elements of one's life constellation, thus sacrificing the realistic classification of reality events for the (temporary) peace of mind. The "defended against" was usually seen as unacceptable drive derivatives or demands and prohibitions of the conscience ("superego"), which had to be covered by amnesia or through other defensive manipulation disguised and thus made "innocuous." A brief clinical vignette may serve as an example:

A man explains that he would like to stay in analysis forever as he likes to learn about himself. Upon analysis this explanation turns out to contain the following additional meanings: the 
analysand can act out his attachment to his parent analyst and can indulge dependency needs; he is afraid of facing the world without the analyst; he is afraid of becoming aware that he, in fact, can face the world on his own, since such attempts in childhood and adolescent seemed connected with a parental loss of love and therefore seems now too threatening. In addition, such continued dependency allows a postponement of coming to terms with certain grandiose childhood fantasies regarding future achievements about which the analysand, by remaining in analysis, imagines that he does not have to be put to the test as yet. All this (and probably more) entered into the analysand's original statement of wanting to continue to learn about himself. Maybe from the way this statement was emphasized during a session, from the way the analysand did not want to question it spontaneously or from the emotional reaction caused by some outsider questioning it, the analyst could sense that anxiety laden issues were latently present and needed to be analyzed. The analysand remained unaware that with his original statement (using the defense of rationalization) he was defending against a fuller awareness of the previously mentioned anxiety laden issues. In the safety of the analytic setting and the help provided by the analyst, these issues could be discussed and, after a working through period, resolved. The regressive tendencies just described were not only operative in this instance but were connected with many other domains of his life. By laying them to rest, a part of personality development which had previously been "frozen" could resume.

Originally, Freud (1900) described defense and defended against in terms of conscious thoughts defending against unconscious ones (topographic theory). At that time cure was conceptualized as the analysand becoming conscious of what was hitherto unconscious. A second stage was reached when Freud discovered that the defenses themselves operated mainly unconsciously, and therefore that the opposing category conscious / unconscious was not the most crucial one for describing the ubiquitous struggle between defense and defended against. He developed what became known as the structural theory (S. Freud, 1923), where an ego system (secondary process thinking; affect) mediates between the id system (drives; primary process thinking), the superego system (norms: demands and prohibitions) and reality. Cure was now defined as strengthening the ego by working through id and superego related material. Defense and defended against was now seen in terms of compromises between these systems. By the working through process, entailing, in the main transference interpretations and genetic reconstructions would allow for more and more flexible, more realistic compromise formations between the opposing claims of these systems could be achieved.

From a classification research point of view the structural theory contained advances as well as deficiencies compared to the topographic theory. Whereas before repression was the only defense of importance, now not repression as such, but the compromise patterns of defensive operations was focused on: the analysand was seen as keeping affects isolated from thoughts (isolation), projecting inner strivings onto others (projection), denying aspects of reality (denial), reversing or repairing fantasied calamities (undoing), escaping responsibility by childlike behavior (regression), escaping becoming aware and dealing with difficult issues by enacting them compulsively (acting out), etc. (A. Freud, 1936.) Each piece of analytic material contains most of the above elements of defenses, combined in a unique way and having a unique meaning.

In a collaborative effort (free association on the part of the analysand, interpretation on the part of the analyst) the epistemologic categories of the analysand change in the direction of greater 
efficiency regarding how the mind works (increase of conceptual parsimony, decrease of anxiety, increase of pleasure, greater freedom to explore new situations, greater tolerance for intimacy).

\section{THE TEMPORAL THEORY}

The need for a new classification (the temporal theory) arose because of theoretical difficulties, including logical inconsistencies in the previous theory as well as new clinical observations (Borbely, 1987).

The basic assumption of the temporal theory is the following: whereas all elements of knowledge, drives, wishes, fears, norms, interests, plans, etc. are organized simultaneously in multiple modes, the two most basic modes are those of two temporal planes: the one reflecting the individual's life cycle, the other the individual's social participation at each biographic moment. The life cycle, or the "vertical temporal plane," includes all stages of personality development from conception to death: genetic endowment, infancy, toddler age, preschool age, latency, adolescence, young adulthood, middle age, etc. The analysis of the succession of these stages reveals the laws of personality development with their progressive and regressive processes. Social participation occurring within the "horizontal plane" represents the individual's relationships and activities at each moment of the life cycle. Depending on the stage in the life cycle as well as on the domain of social involvement of the moment, such social participation may occur as a dyadic relationship with a parent or spouse, as a triadic relationship in the oedipal period or as relationships within the family, peer system or the world of work. While both planes (main semantic systems) each contain an identical set of the previously mentioned elements, these elements are organized differently in each of them, based on the underlying laws operative in the life cycle and the social participatory dimension. At each moment the human mind is both a product of as well as a participant within these two main semantic planes. In mental health these two planes inform each other creatively whereas in neurosis the dialectic relationship between these two planes is disturbed. A sense of relevance as well as anxiety determines the nature of the defenses which mediate the two main semantic systems and within them the semantic subsystems. Through internalizations and (re)externalizations occurring over time, these planes are constituting each other. Personal meaningfulness (relevance within the vertical plane) and personally meaningful social participation (relevance within the horizontal plane) are the governing semantic - pragmatic principles subsuming all other classificatory principles and categories.

The search for relevance is conducted simultaneously within both semantic planes. These planes inform each other and constrain each other by alternating iteratively from a text to a context mode until an overall relevance/anxiety/pleasure equilibrium is established. Many other less essential cycles occur simultaneously within the mind, for example the changing modes of contemplation versus decision, deliberation versus event (Baeckman et al,1986), and objective, lawful processes versus subjective, law designing processes.

In the temporal theory, Borbely views defense and defended against in a new way: defense and defended against are not so much a consequence of anxiety, but rather an expression of the fact that all knowledge, all categories and concepts, all motivational elements have to be organized simultaneously within two different time-related domains, which inform and constrain each other. 
These two domains are organized along criteria of relevance for social participation and the life cycle, respectively. Anxiety and pleasure, in the traditional view of pivotal importance, are seen here as subsidiary to the interplay of these two relevance systems. In the temporal theory the interplay between these two relevance systems can be conceptualized as coping and introspection. These two activities "defend" against each other to the extent that by pursuing one, the other has to, for a brief time, be held in abeyance as if it were an invariant context. This process comes to an end by the focus being reversed. Of course, these two processes occur simultaneously and are separated here only for heuristic purposes. To the extent that anxiety is part of this interplay, the traditional use of "defense" and "defended against", indicating neurotic complication, is in order, as long as one keeps in mind that both sides defend against each other. This view clarifies the clinical fact that defense and defended against are ubiquitous, normally occurring phenomena and not just anxiety induced, neurotic ones. It further explains the clinical observation that anything can serve as defense or anything can be defended against. In the traditional view, the defended against was seen as consisting of childhood drive derivatives, whereas the defenses consisted of thoughts, norms, affects and anxiety. In that anxiety centered view, compromise formations based on coping, introspection, and without anxiety could not be explained. This theoretical shortcoming was obfuscated by the fact that anxiety, to some extent, is usually "imported" from insufficiently resolved childhood conflicts. This means, that even in the absence of symptoms one's creativity can be enhanced by deeper knowledge about one's self and one's interactions with the world.

While the two basic organizational modes contain similar elements of "knowledge atoms" they differ in their basic temporal significance: the "vertical" plane with its past/future dimension represents an ontogenetic text/context, whereas the "horizontal" plane with its emphasis on the present represents an "actual - genetic text/context. Dynamically viewed, all of the individual's codifications of past experience influencing the presently occurring social participation can be called "transference" (transferred from the past) and all influence on previous codifications stemming from presently occurring social participation can be called "inference" (Borbely, 1980). Transference and inference are psychological forces dialectically related to each other and constantly changing into each other over time. They represent experiential forces underlying the individual's changing classification system ("priorities"). "Transference", as the unconscious dramatization of feelings, attitudes and identifications is one of the key concepts of psychoanalysis. "Inference", as the force informed by presently occurring social participation leading to changes within the personality's previous codifications, was not previously conceptualized in psychoanalysis. Within computer science, inference is conceptualized, albeit usually without its feedback function for changing previous premisses. It can be hoped, that in the future both psychoanalysis as well as the computer sciences take full cognisance of these two dialectically related forces.

Underlying the basic assumption described above are the following creativity related notions: the individual is a unique entity of semantic processing endowed with creativity (generating new meanings, new categories, new classifications, new insights, new sciences, new technologies, etc.). The individual, in his or her social participatory dimension, is connected with the collective, historic processes of mankind. The latter has to be seen as another entity, where semantic processing endowed with creativity occurs. These two entities (the individual and society) differ, of course, as to their lawful and law-designing patterns, which nevertheless are, at the same time, dialectically related to each other. Here we are only focussing on the individual's partaking of 
humanity's progress via his or her social participation. Through such social participation the individual receives love, knowledge, categories of thinking, classifications for such categories, task requirements and problem formulations leading to problem solving and new problem posing activities involving ever new classifications. Through selective internalization of such social participation the individual's need structure, including all internal classifications, change continuously.

\section{THE TEMPORAL THEORY AND SOME CONCEPTS WITHIN INFORMATION SCIENCE:}

\section{a) Parallel Processing:}

The semantic main planes as well as the semantic subplanes can be thought of as processors processing the same experiential events according to differing criteria. All experiential events have to be processed according to their life-cycle and social-participatory relevance. Within these, all events have implications for one's corporeal relations, interpersonal relations and social relations and have to be processed simultaneously according to these criteria.

\section{b) Distributive Processing:}

The concepts in all branches of the semantic trees change continuously, due to the dialectic between concept change leading to semantic organization changes as well as semantic organization change leading to concept change. These transconceptual, transclassificational changes can today best be conceptualized by theories of parallel distributed processing (McClelland,J.L. et al, 1985).

\section{c) Hybrid Reasoning:}

The human brain employs many forms of rationality but, especially in the less conscious parts of the mind, it uses so called primary process thinking, a form of illogical, primitive thinking underlying much of conscious thought, art, dreams, creativity, etc. The most varied forms of self expression, like thoughts, affects, drive derivatives, dreams, amnesias, sudden interruptions in communication, the switching to a different subject, body language, and many more are simultaneously considered and integrated by the analyst in order to understand "what's going on," what the analysand is struggling with at the moment. The interplay between all these forms of thought, enriched and constrained by emotions and drives, represents what in computer science is called hybrid reasoning.

\section{d) Information Retrieval:}

In psychoanalysis, information retrieval starts with an anamnesis. The analyst knows that important information has fallen pray to amnesia, to anxiety induced repression. This information will reappear, slowly, either reenacted in the transference or, after anxiety reduction followed by repression removal, directly remembered. Only after some of the dynamically important categories have been worked through, in other words reclassified, can further relevant information retrieval take place. Information retrieval itself is governed by presently important relevance considerations and therefore has to be seen as an active, constructive process. 


\section{e) Compatibility between Classification Schemes:}

The psychoanalyst has to deal with the sequelae of classification schemes stemming from different developmental stages. The infant's, the toddler's, the preschool child's classification is not only each different in a quantitative or qualitative sense but is also organized differently. All these classification schemes differ from adult ones. Within the adult classification schemes we have already encountered the temporal main planes and the different semantic subplanes. Depending on the task at hand, one classification scheme will take a central position relegating others to the periphery. For example, during love making, corporeal relations achieve temporary supremacy over interpersonal or social relations. During dating interpersonal relations, during a club meeting social relations might be in the foreground. In many cases several such semantic subdomains will be simultaneously active to varying degrees, enriching and constraining each other. It is the mark of an experienced and talented analyst to be able to simultaneously receive communications along all these classification scheme channels (Tulving, E. 1985.), integrate them (mainly unconsciously) and communicate them back to the analysand in a new and meaningful way

\section{f) Knowledge Representation Schemes:}

As long as the neurotic conflicts persist, both the conscious as well as the unconscious knowledge representations pertaining to the conflict, but also those not directly pertaining to them, will be more or less skewed. Also, during normal growth processes, understood as classification changes in the most basic sense, the knowledge representation schemes undergo continuous change. A clinical vignette may serve as an example: A young man reveals to the analyst his conviction of being "a bad boy." After some analysis this fantasy, active already in childhood, could be understood as being connected with intense anger towards the parents, which in turn had to do with a perceived parental rejection. Such anger had, for the most part, been repressed so as not to threaten the love and safety needs of the child. To the extent that it was expressed, the child had been made to understand that he was "a bad boy." To be bad also meant the hope of finding some attention where not enough was given and to avoid the painful feeling of rejection. It further turned out, that what was felt as rejection had to do with the parents feeling overwhelmed with their own life as well as with their intolerance for accepting autonomy in their children.

This vignette should highlight how "knowledge" gets represented based on existing classification schemes and later re-represented based on newly acquired classification schemes. Knowledge is shown here as dependent on the experiential quality and depth. The latter itself is based on the need structures of the experiencing person as well as on the ones of important other subjects representing the context of the experience. To utilize previous knowledge structures often involves changing their previous meaning. Such a change, in turn, will influence the knowledge representation scheme in the present which gave rise to the exploration of the one in the past. In order to temporarily interrupt this cycle, a decision mechanism is called for, exposing a conclusion to the interaction with collective processes. Defense and defended against represent such a temporary conclusion, a result of "reasoning with uncertainty," which entails always a measure of repression and denial. While in the traditional view, anxiety lead to repression and denial, the temporal theory assumes that the necessity to come to conclusions and decisions in the context of intrasystemic and 
extrasystemic uncertainty (which often is connected with anxiety) entails unavoidably a cutting off of any further exploration, which then gets expressed as repression and denial.

\section{RECENT HISTORIC CHANGES AND CLASSIFCIATION RESEARCH:}

Classification research currently suffers from a lack of theories explaining the dynamics underlying change in the hierarchies of classification systems (Anderson, 1983). The need for understanding the laws of these dynamics arises at a time of unprecedented changes accompanying the scientific - technological revolution leading from the industrial to the information age. A profound redefinition of most previously stable classification schemes accompanies this transition. Creativity, previously reserved for human beings, is now envisaged for machines. Socialism, originally defined for the industrial age, undergoes a profound crisis and redefinition trying to find its bearings in the information age. Science, accustomed to discovering natural laws begins to design its own laws, as for example in the fields of programming languages, computer assisted software development, genetic engineering, etc. The change from the industrial age to the information age entails moving from production (matter as substance and energy) to creation (matter as information).

Classification, underlying all human endeavors, changes over time according to subject specific dynamics. Therefore, a focus on categories alone (e.g., Rosch, 1973), while as such valuable, remains insufficient for the task of comprehending what underlies changes in categories and classifications. Without a theory of psychodynamics and sociodynamics underlying category change within the individual and the collectivity, as well as a theory of their interaction, category research will be severely constrained.

In this paper the individual and mankind as a whole were mentioned as subjects endowed with semantic processing on the level of creativity, that is, where category change occurs in the context of potential authenticity (self growth) and progress (science and culture). The question whether other similarly endowed entities exist, e.g. the family, computers, etc. was not here addressed.

In the individual, both the acquisition of external information as well as its integration with internal information is mediated by how the individual experiences it. The category of "experience" can therefore not be neglected. Essentially, each psychologically growing individual creates his or her own distinctive classification scheme. The uniqueness of an individual's biography entails a uniqueness of that individual's patterns of change in classification schemes. To the extent that such uniqueness is associated with authenticity, love, inventiveness, organizational abilities, etc., the individual can be said to be creative. Therefore, research about classification change is intimately connected with research about creativity, where the question of the relationship of less creative versus more creative processes to classification change could be elucidated (the classificatory significance of paradigm changes.)

Psychoanalysis has shown that one's past development has not once and for all occurred. It is subject to reinterpretation and can be changed regarding its effects on the present. As such, psychoanalysis became one of the first sciences with law-designing, not only law-discovering 
potential, one of the first sciences focusing not on nature (production as intercourse between man and nature) but on information (creativity as intercourse between the new and the old).

\section{BIBLIOGRAPHY}

Anderson, J.R. 1983. The architecture of cognition. Harvard Univ. Press.

Baekman,L., Nilsson, L.-G., Chalom,D. 1986. New evidence on the nature of the encoding event. Memory and Cognition, 14:4 339-346.

Borbely, A.F. 1980. The Dialectical Unity of Transference and Inference in Psychological Development. Interaction 3:4 199-207. 1989.a) Towards a Temporal Theory of the Mind. Psychoanalysis and Contemporary Thought 10:4 459-487. 1989.b) Der Realitaetsbegriff der Psychoanalyse oder Psychoanalyse im Uebergang. Jahrbuch fuer Psychopathologie und Psychotherapie IX/1989 91-113, Pahl - Rugenstein Verlag.

Brenner, C. 1982. The Mind in Conflict. International Universities Press.

Freud, S. 1900. The interpretation of dreams. The Standard Edition of the Complete Psychological Works of Sigmund Freud. Hogarth Press, 1962. 1923. The ego and the id. The Standard Edition of the Complete Psychological Works of Sigmund Freud. The Hogarth Press, 1962.

Freud, A. 1936. The Ego and the Mechanisms of Defense. New York International Universities Press, 1946.

Frisch, A. M. 1991. The Substitutional Framework for Sorted Deduction: Fundamental Results on Hybrid Reasoning. Artificial Intelligence 49:161-198.

McClelland, J.L.; Rumelhart, D.E. (1985) Distributed memory and the representation of general and specific information. Jl. of exp. Psych: General 114, 159-188.

Rosch, E. 1973. On the internal structure of perceptual and semantic categories. In T.E. Moore (ed), Cognitive development and the acquisition of language. New York: Academic Press.

Tulving, E. 1985. How many memory systems are there? American Psychologist, 40: 385-398. 
Borbely, A. (1991). The Semantic Classification in the Temporal Theory of Psychoanalysis. 2nd ASIS SIG/CR Classification Research Workshop, 31-40. doi:10.7152/acro.v2i1.12545 